\title{
Performance Degradation of Existing Coastal Reinforced Concrete Bridges and Prediction on Remaining Service Life
}

\author{
Xinyi Chen \\ Department of Mechanical, Chemical and Materials Engineering, University of Cagliari, Cagliari, Italy
}

\begin{abstract}
Compared with the roads and reinforced concrete bridges inland, roads and bridges in coastal region is easier to be destroyed which makes its durability lower because of chloride ion erosion. Steel structures in coastal roads and bridges have been corroding by chloride ion for a long time which caused fast collapse after rotten and aged steel appeared. For coastal region, therefore, every year, a large amount of money is used to reinforce and maintain the roads and bridges, which brings serious economic losses and in a way affects the social environment in coastal region.
\end{abstract}

Keywords: Coastal Region; Concrete; Steel; Remaining Service Life.

\section{EVALUATION CRITERIA FOR THE BRIDGE'S REMAINING SERVICE LIFE}

From a scientific point of view, the key point to determine the bridge's service life lies in the degree of corrosion and rust of the concrete reinforcement, and the carbonization degree of the concrete itself [1]. Only having an objective judgment of these factors can we effectively determine the bridge's remaining service life [2]. However, since the bridge's reinforcing bar and concrete show very complex corrosion reaction, the current judgment methods for the bridge's remaining service life are not the same. The following several judgment methods are more commonly used evaluation criteria for the bridge's remaining service life in the current bridge engineering maintenance.

1.1 Evaluation criteria for carbonation service life.

Evaluation criteria for carbonation service life, as the name suggests, are to take concrete carbonization as the main basis for determination. Concrete carbonation leads to the loss of reinforcement protection, so for the evaluation criteria for carbonation service life, the complete loss of concrete reinforcement protection, followed by initial corrosion of steel, is taken as the starting point of the bridge structure's remaining service life [3].

1.2 Evaluation criteria for corrosion cracking service life.

The core of evaluation criteria for corrosion cracking service life are to take the time at which the concrete appears corrosion-induced crack along the steel as the starting point of the bridge structure's remaining service life. Because for the coastal bridge, once the concrete as a protective layer appears corrosion-induced crack along the steel, it means that the steel wrapped in the concrete has to face the corrosion of chloride ions alone, and then the decay of steel will speed up, which is bound to seriously affect the bridge's remaining service life. Therefore, in the process of bridge maintenance, concrete corrosion-induced crack along the steel is often regarded as the safety limit of reinforced concrete [2] [3].

1.3 Evaluation criteria for bearing capacity service life.

Evaluation criterion for bearing capacity service life is a kind of remaining service life prediction method based on bridge structure with steel corrosion as the criterion. Because for the bridge steel, facing the corrosion of chloride ions means that its internal structure has shown a very unstable state. In this case, the bearing capacity of steel will continue to decline, its durability will also gradually get worse. As a result, the current bearing capacity of the steel along with its future bearing capacity life is actually the bridge's remaining service life [3].

\subsection{Steel Corrosion.}

In fact, for infrastructures in coastal region, steel corrosion is the primary factor affecting remaining service life of roads and bridges. Generally speaking, compared with concrete, steel is the crucial foundation to support roads and bridges. Their remaining service life is definitely and greatly shortened due to primary support for roads and bridges do not exist if steel structures are destroyed. However, lots of mathematical models are absolutely useless to provide improvement measures to solve problems of steel corrosion, although relative researches on durability of reinforced concrete in China and other countries is too numerous to count. Therefore, we have more work to do in e future [2] [4]. 


\section{SHORTAGES IN PRESENT STUDY ON REMAINING SERVICE LIFE OF CONCRETE BRIDGES}

2.1 Shortages of service life Prediction Based on Durability.

Firstly, in fact, with the present situation, a large number of researches and analysis with qualitative and quantitative perspectives on problem of concrete carbonization have been carried out in China or other countries. On the whole, however, by reviewing the researches, serious shortages exist in the researches concerning about corrosion effect of chloridion on steel. In other words, the present researches on service life of concrete bridges still focus on concrete carbonization as a superficial phenomenon. The core problems have not been researched and steel corrosion should be analyzed further. Secondly, for concrete bridges, stability and bearing capacity for concrete structure are deciding factors for safety of concrete bridges. Researches on concrete bridges, therefore, have to in detail study and analyze the durability of concrete materials in addition to concrete carbonization. Only by this way can service life of concrete bridges be prolonged actually. Unfortunately, respects to this point, many shortages exist in researches on concrete bridges in China and other countries. Thirdly, for concrete bridges, a series of indexes of durability of concrete bridges are changed along with time variation. Therefore, reparation and maintaining are of deciding factors that prolong service life of concrete bridges. Furthermore, based on reparation and maintaining, the potential hidden danger and remaining service life can be recognized clearly and controlled firmly, which is convenient for constructors to make preparations in advance for reconstruction of concrete bridges. Considering the word nowadays, lots of researches attach importance to increase durability of concrete bridges from perspective of original materials. Few of researches about theories of reparation and maintaining and model building were issued. They are the reasons why shortages in researches on remaining service life exist.

\subsection{Structural Resistance Model and Remaining service life Prediction Based on Material Degradation.}

1) Detection for Chlorineion Content:

For concrete bridges in coastal region, chlorine ion content if the key factor affecting durability of concrete bridges. Detection for chlorine ion content, therefore, can be applied in predicting remaining service life of concrete bridges. By RTC, as known as rapid chlorideion tester, chlorideion content in existing concrete bridge can be obtained clearly. According to calculation methods given by relative data and Loading Test and Structural Evaluation for Roads and Bridges, it is convenient to presume and get diffusion coefficient of chlorideion in concrete, which is helpful to predict the remaining service life of concrete bridges.

2) Service Life Prediction for Typical Bridge Durability:

In the methods used to predict service life of typical bridges based on durability, factors that include concrete carbonization and steel corrosion etc are key indexes to measure remaining service life of concrete bridges. It is a worse signal if concrete cover is peeled off or steel is corroded seriously. It shows that remaining service life of concrete bridge is not long and the bridge should be repaired and maintained as soon as possible. At present, in China or other countries, researchers in a way comprehensively understand the concrete carbonization, which makes it possible to predict service life of concrete bridges based on durability in coastal region depending on concrete carbonization.

\section{CONCLUSIONS}

Based on the study mentioned above, what decides the remaining service life of concrete bridges in coastal region is degree of concrete carbonization and steel corrosion. Presently, obvious shortages exist in researches on steel corrosion although researches and theoretical basis about process of concrete carbonization are mature in China and other countries. This is what the researches and prediction about service life of concrete bridges in coastal region should pay attention to. It is quite significant for social stability in coastal region that constructors predict performance degradation and remaining service life of concrete bridges. Mastering the rules of remaining service life of concrete bridges in coastal region scientifically is the only way for us to keep a normal use of concrete bridges, bring welfare to society and the public.

\section{REFERENCES}

[1] China Concrete Institute. Prediction the Remaining Useful Life and Study of Performance Degaration for R.C Bridge Near Coastal Areas. BeiJing: China Science and Technology Culture Press, 2015.

[2] Ju Liyan; Zhang Xiong, "Study on Performance Degeneration and Remaining Service Life of Existing Armored Concrete Bridge," Construction Technology, 2013.

[3] Hua Yuan, Zhang Shaobo, Jiang Zhiqing, "Study on Performance Degeneration and Remaining Service Life of Existing Armored Concrete Bridge Near Coastal Areas," China Concrete and Cement Products, 2014.

[4] Li Yongqiang; Che Huiming, "Analysis on Performance Degeneration of Existing Armored Concrete Bridge," Journal of the China Railway Society, 2015. 